\title{
Eupatilin Content and HPLC Fingerprints of Fresh Leaves of Artemisia argyi from 52 Germplasm Resources
}

\author{
Hankui Wu ${ }^{1,}$ *, Xingqiang Gao ${ }^{1}$, Xinyong You ${ }^{1}$, Jianguang Chen ${ }^{1}$, Zhen $\mathrm{Su}^{1}$, Yuanping Wang ${ }^{1}$, \\ Kunpeng Zhang ${ }^{1, *}$, Linlin Hou ${ }^{2, *}$ \\ ${ }^{1}$ School of Biotechnology and Food Engineering, Anyang Institute of Technology, Anyang, China \\ ${ }^{2}$ School of Chemistry and Chemical Engineering, Anyang Normal University, Anyang, China \\ Email address: \\ wuhankui222@126.com (Hankui Wu),1095557379@qq.com (Kunpeng Zhang), linlin9918@163.com (Linlin Hou) \\ ${ }^{*}$ Corresponding author
}

\section{To cite this article:}

Hankui Wu, Xingqiang Gao, Xinyong You, Jianguang Chen, Zhen Su, Yuanping Wang, Kunpeng Zhang, Linlin Hou. Eupatilin Content and HPLC Fingerprints of Fresh Leaves of Artemisia argyi from 52 Germplasm Resources. International Journal of Chinese Medicine.

Vol. 5, No. 3, 2021, pp. 38-47. doi: 10.11648/j.ijcm.20210503.11

Received: July 9, 2021; Accepted: July 19, 2021; Published: July 24, 2021

\begin{abstract}
Artemisia argyi is a well-known medicinal plant which has been used in traditional Chinese medicine for thousands of years. It is usually named Aicao or Aihao in China, and the leaves are processed and applied in moxibustion to treat different kinds of diseases. In order to select the best germplasm resource to grow high quality herbal products, we have compared the HPLC fingerprints profiles of 52 geographically different germplasm resources collected from 17 provinces of China. The results data were processed by similarity and hierarchical clustering analyses. In addition, common patterns of a typical local variety of $A$. argyi sourced in Henan province are established, and sample similarities were evaluated by selection of 14 typical chromatographic peaks of 10 samples and are found to vary between 0.664 and 0.969 . The results show that the HPLC fngerprint analysis and quantitative analysis is a powerful tool to identify and control the quality of fresh $A$. argyi and related products. In addition, the content of a major secondary metabolite in the leaves of $A$. argyi known as eupatilin, which is a flavone with numerous bioactivities such as anticancer, anti-inflammation and anti ulcer has been determined for all the 52 samples by HPLC-DAD. The results provided great scientific basis for the research and development of the herb as popular products for the health care of human beings.
\end{abstract}

Keywords: HPLC, Fingerprints, Eupatilin, Content

\section{Introduction}

The medicinal plant Artemisia argyi Levl. et Vant. is quite famous and popular in China and worldwide [1]. (Figure 1). It is edible and can be used to make pastries, breads, dumplings, cakes, and can be mixed with rice or processed into tea or wine. It has also been used as an air purifier and a mosquito repellent [2]. A. argyi is widely distributed in Korea, Mongolia, Japan, and the Russia Far East [3]. Besides its application in moxibustion, it was claimed that $A$. argyi could treat over one hundred diseases in ancient Chinese books such as Compendium of Materia Medica (Bencao gangmu) and Formularies for Fifty Two Kinds of Disorders (52 Bing Fang). There are four famous $A$. argyi recorded in China, namely northern $A$. argyi (produced in Tangyin, Henan), Hai
A. argyi (from Ningbo, Zhejiang), Qichun $A$. argyi (from Qichuan, Hubei) and Qi A. argyi (from Anguo, Hebei), which are known as genuine herbs of high quality [4].

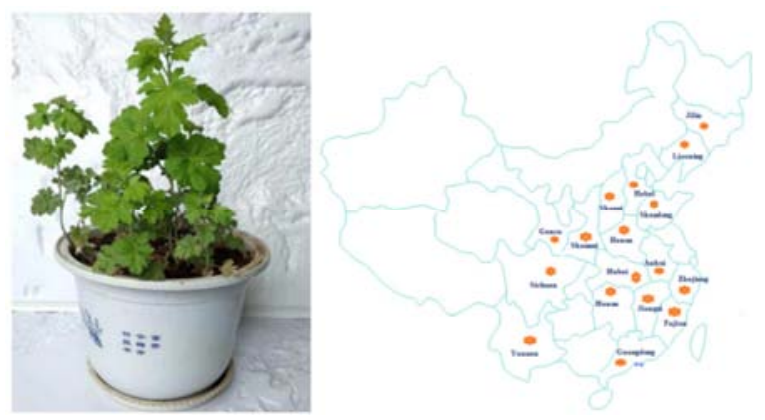

Figure 1. Northern A. argyi and germplasm resources of 52 samples. 
The Chinese Pharmacopoeia (almost each edition) has recorded that $A$. argyi folium is the dry leaves of $A$. argyi. It can be used to warm meridian to treat blood related diseases, to disperse cold to relieve pain, and to dispel dampness and stop itching [5]. The chemistry, pharmacology and quality control methods of $A$. argyi have been studied thoroughly and summarized in recent years [6]. The plant contains complex ingredients, including essential oils, polysaccharides, flavonoids, tannins, sterols, and terpenes [7]. The leaves of $A$. argyi are often utilized to prevent and cure gynecological, respiratory, and dermatological diseases, such as menstruation-related symptoms, infertility, dysmenorrhea, inflammation, hemostasis, tuberculosis, asthma, and eczema [8-10]. However, geographically different origins of $A$. argyi might vary in chemical compositions and biological functions. The international standard ISO 20759-2017 has been established for the dry leaves of $A$. argyi, which only specifies the minimum requirements and test methods of dry and processed $A$. argyi leaves for medicinal use and is suitable for identification and quality control of this herbal medicine. There have been plenty of studies on the chemical or chromatographic fingerprints of dry materials of $A$. argyi in rencent years [11-15]. Nevertheless, there is no report found on the quality analysis of fresh leaves of $A$. argyi. It is necessary to establish a certain standard for the fresh raw materials of the germplasm resources of $A$. argyi to ensure the final products made from it are qualified. Unfortunately, identification of the plant is quite difficult because it has very similar morphological features to related species, such as $A$. lavandulifolia, A. princeps, and A. stolonifera. The HPLC fingerprinting has an entirety and fuzziness feature when coupled with quantitative analysis, which is a comprehensive and quantifiable identification method and has been used for a couple of years to evaluate the quality of herbal products [16]. WHO has adopted this method for assessing the quality of herbal products owing to its convenience and efficiency [17].

In order to reveal the chemical variations of fresh leaves of A. argyi origin from different locations, we have collected 52 germplasm resources from 17 provinces of China (Figure 1 and table 1), which are subsequently grew in our culture laboratory. Herein, an effective HPLC fingerprinting method coupled with hierarchical clustering analysis (HCA) and principal component analysis (PCA) is established for the identification and quality evaluation of fresh $A$. argyi from different origins. This method allows us to comprehensively profile the chemical composition and similarities of $A$. argyi from different locations, which are of great value for the further study and development of this medicinal plant.

Table 1. The locations of plant materials collected from different regions and the content of eupatilin.

\begin{tabular}{|c|c|c|}
\hline No. & Origin & Eupatilin $(\mu \mathrm{g} / \mathrm{g})$ \\
\hline $\mathrm{S} 1$ & Yantai, Shandong & 3.64 \\
\hline $\mathrm{S} 2$ & Jinan, Shandong & 7.35 \\
\hline S3 & Xingtai, Hebei & 3.76 \\
\hline S4 & Zhoukou, Henan & 4.39 \\
\hline S5 & Zhengyang, Zhumadian, Henan & 6.22 \\
\hline S6 & Kunming, Yuannan & 3.75 \\
\hline S7 & Zhaojiahe, Anyang, Henan & 3.85 \\
\hline S8 & Yuxi, Yunnan & 3.82 \\
\hline S9 & Yibin, Sichuan & 3.78 \\
\hline S10 & Yuzhou, Xuchang, Henan & 3.65 \\
\hline S11 & Gaoxinqu, Luoyang, Henan & 4.44 \\
\hline $\mathrm{S} 12$ & Huangshi, Hubei & 6.38 \\
\hline $\mathrm{S} 13$ & Nanyang, Henan & 5.77 \\
\hline S14 & Jinzhai, Anhui & 5.57 \\
\hline S15 & Kaifaqu, Anyang, Henan & 3.83 \\
\hline S16 & Hezi, Shangdong & 7.21 \\
\hline S17 & Ruyang, Luoyang, Henan & 4.22 \\
\hline S18 & Shaxian, Fujian & 3.89 \\
\hline S19 & Huizhou, Guangdong & 3.87 \\
\hline S20 & Anguo, Hebei & 5.06 \\
\hline $\mathrm{S} 21$ & Poyang, Jiangxi & 6.84 \\
\hline $\mathrm{S} 22$ & Linzhou, Anyang, Henan & 3.95 \\
\hline S23 & Neixiang, Nanyang, Henan & 3.76 \\
\hline S24 & Xiangyang, Hubei & 6.75 \\
\hline $\mathrm{S} 25$ & Shaoyang, Hunan & 8.38 \\
\hline S26 & Puyang, Henan & 27.64 \\
\hline
\end{tabular}


Table 1. Continued.

\begin{tabular}{|c|c|c|}
\hline No. & Origin & Eupatilin $(\mu \mathrm{g} / \mathrm{g})$ \\
\hline S27 & Nanyang, Henan & 5.02 \\
\hline S28 & Neihuang, Anyang, Henan & 4.61 \\
\hline S29 & Hengshui, Hebei & 4.76 \\
\hline S30 & Queshan, Zhumadian, Henan & 4.71 \\
\hline S31 & Fuzhou, Fujian & 5.13 \\
\hline S32 & Quyang, Hebei & 3.90 \\
\hline S33 & Changzhi, Shanxi & 3.95 \\
\hline S34 & Qichun, Hubei & 5.94 \\
\hline S35 & Chengdu, Sichuan & 3.79 \\
\hline S36 & Quzhou, Zhejing & 10.16 \\
\hline S37 & Zhaolinqu, Luohe, Henan & 3.79 \\
\hline S38 & Jibei, Hengshui, Hebei & 4.68 \\
\hline S39 & Tangyin, Anyang, Henan & 5.00 \\
\hline S40 & Luohe, Henan & 4.11 \\
\hline S41 & Guandu, Kunming, Yunnan & 4.27 \\
\hline S42 & Taigu, Shanxi & 4.52 \\
\hline S43 & Linjiang, Jilin & 5.35 \\
\hline S44 & Dali, Yunnan & 3.69 \\
\hline S45 & Luzhou, Sichuan & 3.50 \\
\hline S46 & Changge, Xuchang, Henan & 5.43 \\
\hline S47 & Mianyang, Sichuan & 5.81 \\
\hline S48 & Xi'an, Shannxi & 5.85 \\
\hline S49 & Dingxi, Gansu & 5.00 \\
\hline S50 & Fushun, Liaoning & 4.64 \\
\hline S51 & Hengdian, Zhejiang & 7.45 \\
\hline S52 & Ganzhou, Jiangxi & 6.38 \\
\hline
\end{tabular}

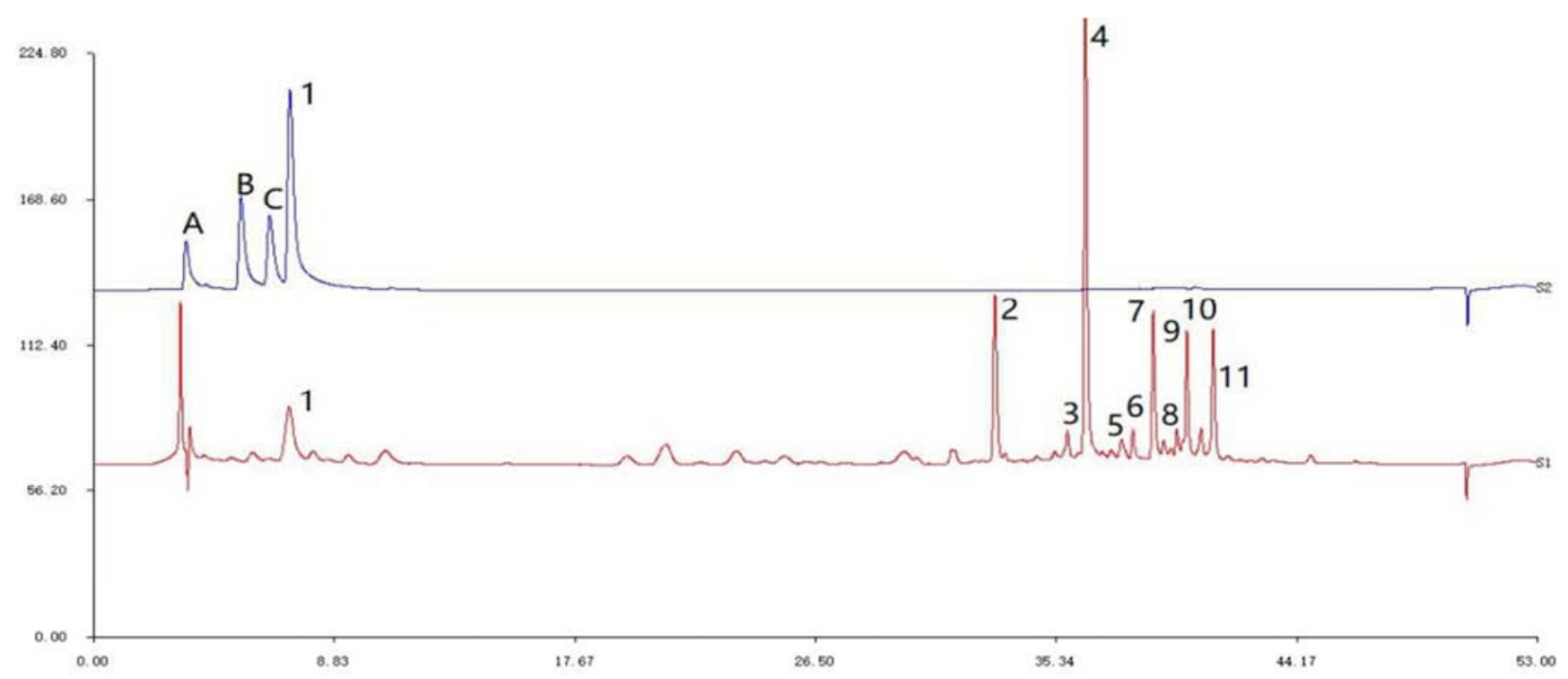

Figure 2. Position of of the peaks of four reference compounds. 


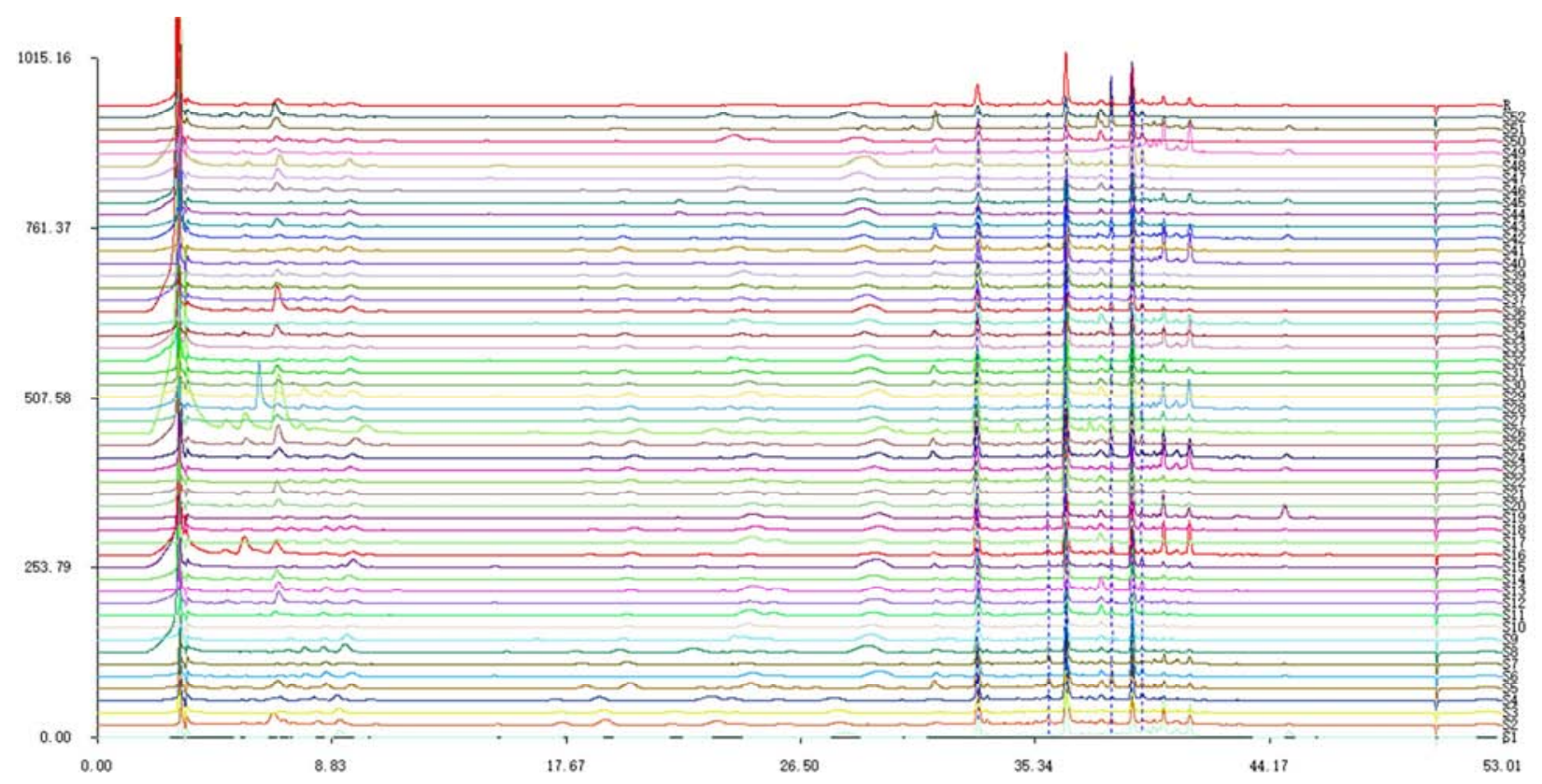

Figure 3. HPLC fingerprints of 52 fresh A. argyi samples (S1-52, Table 1) and the reference fngerprint (R) obtained by the Similarity Evaluation System.

\section{Experimental}

\subsection{Plant Materials}

Fifty-two germplasm resources (S1-52) of $A$. argyi were collected from 17 different provinces (Table 1) in China. The authors declared that all the methods were carried out in compliance with the Regulations of the People's Republic of China on the Protection of Wild Plants. Subsequently, the plants were grown in the culture room of our laboratory, with controlled light, temperature and humidity. All samples were identified by the co-author Dr. X. G., because he focused on a project "Collection, classification and evaluation of germplasm resources of A. argyi." (Code: YPY2020028) supported from Anyang Institute of Technology and the specimens were kept in the herbarium of this institute.

\subsection{Chemical Reagents and References}

HPLC-grade ethanol was acquired from aladdin (Shanghai, China). pure water (Wahaha, Hangzhou, China) was bought from a local supermarket. Analytical grade ethanol was purchased from Sinopharm Chemical Reagent Co., Ltd. (Shanghai, China).

\subsection{Standard Solution Preparation}

Rutin, apigenin, jaceosidin, and eupatilin ( $>98 \%$, as determined by the HPLC area normalization method) were obtained from Pufei De Biotech Co., Ltd. (Chengdu, China). The reference compounds were accurately weighed, dissolved in ethanol, and diluted to appropriate concentrations for calibration curve establishment. All stock and working standard solutions were stored in a refrigerator at $4^{\circ} \mathrm{C}$ before use.

\subsection{Sample Solution Preparation}

Fresh leaves of $A$. argyi were cut from the plants. Different extraction solvents (ethanol and $70 \mathrm{vol} \%$ aqueous ethanol) and numbers of extractions $(1,2$, and 3$)$ were measured for extraction optimization. On the basis of a preliminary test, a $1.0 \mathrm{~g}$ fresh sample was extracted with ethanol $(10 \mathrm{~mL})$ for 48 $\mathrm{h}$ at room temperature. The extract was filtered through a $0.45-\mu \mathrm{m}$ Nylon filter and subjected to HPLC analysis (injection volume $=10 \mu \mathrm{L}$ ).

\subsection{Instrumentation and Chromatographic Conditions}

HPLC analysis was carried out on an Agilent 1260 HPLC system (Agilent Technologies, Inc., Santa Clara, CA, USA), which was equipped with a quaternary pump, an autosampler, a degasser, an automatic thermostatic column compartment, a diode-array detector, and a computer with Chemstation software used for HPLC data analysis. A YMC-ODS C18 reversed-phase column $(5 \mu \mathrm{m}, 250 \mathrm{~mm} \times 4.6 \mathrm{~mm})$ was used for separation, with the column temperature set to $35^{\circ} \mathrm{C}$. The following elution system comprising water (phase A) and ethanol (phase B) was used: $0-25 \mathrm{~min}, 45-65 \% \mathrm{~B} ; 25-35$ min, $65-100 \%$ B; 35-45 min, $100 \%$ B; $45-48 \mathrm{~min}, 100-45 \%$ $\mathrm{B}$; 48-53 min, $45 \% \mathrm{~B}$. Absorbance was monitored at $350 \mathrm{~nm}$, the mobile phase flow rate was set at $0.8 \mathrm{~mL} / \mathrm{min}$, and on-line UV spectra were recorded in the range of 190-700 $\mathrm{nm}$. The injection volume equaled $10 \mu \mathrm{L}$.

\subsection{Method Validation}

The method was validated according to Lu et al. [16] for repeatability, precision, stability, and accuracy following the International Conference on Harmonization guidelines. Repeatability was assessed by analyses of five independently prepared extracts of $A$. argyi samples. Precision was 
evaluated by injecting the same sample solution five times within 24 hours. The stability test was performed by injecting the identical sample $0,2,4,6,12$, and $24 \mathrm{~h}$ after preparation. During this period, the solution was kept at room temperature. For the recovery test, in order to evaluate accuracy, a $1.0-\mathrm{g}$ fresh sample of $A$. argyi was independently weighed five times and spiked with a known amount of reference using the corresponding standard. The spiked samples were extracted and quantified as outlined above.

\subsection{Data Analysis}

For typical HPLC fingerprint establishment, all samples were analyzed using the presented methods, and the obtained data were exported in AIA format and imported into a professional software named Computer-Aided Similarity Evaluation System for Chromatographic Fingerprint of Traditional Chinese Medicine (China Committee of Pharmacopeia, 2004A) [17]. This system is very useful to determine the similarity of the chemical composition of different samples. PCA and HCA were performed for pre-standardized data using the Unscrambler $\mathrm{X}$ software package (version 10.2). Components were supposed to draw the PCA scatter plot. The average-linkage-between-groups method was then applied for HCA, with square Euclidean distance used to establish the distance matrix between observations.

\section{Results and Discussion}

\subsection{Extraction of Fresh Leaves}

The leaves of $A$. argyi were chosen because the moxibustion application of this plant is leaves, and volatile compounds contribute to most of the functions of moxibustion. Secondary metabolites were often found in glandular trichomes on the leaves [18]. So there is no need to crush raw materials just for a rough comparison. Because of the same growing conditions in our culture laboratory, the fresh leaves have nearly the same water content. Therefore, a drying step is also not required for fast evaluation to minimize the decomposition of the compounds and lost of volatile substances. The solvent selected for the extraction should be in line with the concept of green chemistry and dissolve compounds of middle to low polarity, and the extraction temperature should be considered as the chemical structures of compounds might be thermally unstable. As a result, ethanol was chose to fulfil the extraction process at room temperature. The fresh samples were soaked in pure ethanol within 30 min just after cutting from the herb, and the extraction time is 48 hours at room temperature.

\subsection{HPLC Condition Optimization}

The HPLC column, detection wavelength, flow rate, column temperature and mobile phase elution procedure have been optimized to get the best separation of all peaks in the fingerprint chromatograms of $A$. argyi in a relative short time. A full-scan experiment data of four reference compounds as rutin, apigenin, jaceosidin, and eupatilin by photo-diode array detection at 220 , 254, $350 \mathrm{~nm}$ showed that the weavelenth of $350 \mathrm{~nm}$ can maximize the number and resolution of all marker compound peaks with satisfactory baseline separation. For resolution improvement, the column temperature $\left(30,35\right.$ or $\left.40^{\circ} \mathrm{C}\right)$ and mobile phase flow rate were optimized, and the best peak separation and shape were obtained at $35^{\circ} \mathrm{C}$ and $0.8 \mathrm{~mL} / \mathrm{min}$ when using ethanol-water as mobile phase. With the idea of green solvents in mind, we decided to use ethanol-water system as mobile phase, which could achieve satisfactory separation, although methanol-water and acetonitrile-water with different modifiers might be better. For the gradient optimization, we have tested different gradient time and mobile phase compositions, the best separation was attained within $53 \mathrm{~min}$ using the optimized procedure. Because the ethanol extract of the fresh leaves has a lot of hydrophobic compounds, the $100 \%$ ethanol elution for a relative long period is necessary to reduce irreversible retention of compounds in the column.

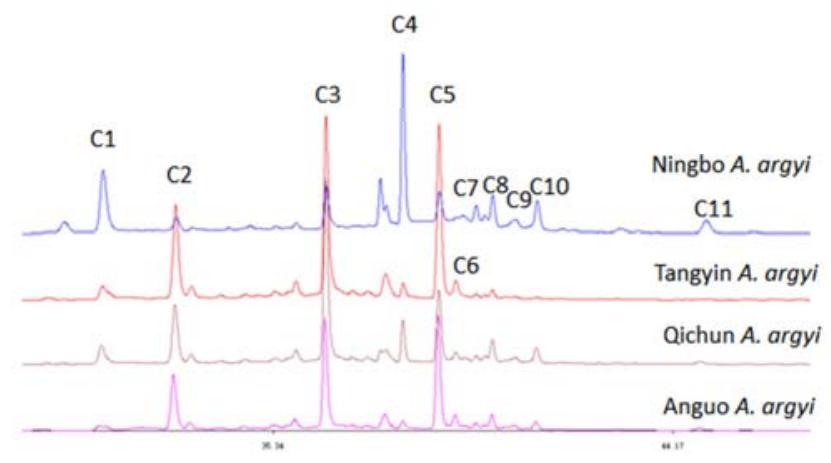

Figure 4. HPLC fingerprint of "four famous A. argyi" from retention time 30-48 $\min ($ part $C$ ).

\subsection{Validation of the Analysis Method}

The precision, stability, and reproducibility were validated, and a well-linear relationship between the peak area and concentration of each reference compound $(\mathrm{R}>0.995)$ was observed for all analytes within the test range. To measure the precision of this method, sample 1 (S1) of $A$. argyi was extracted, and then injected into HPLC for analysis 5 times in 1 day. Stability was determined by injection of the same sample solution in $0,2,4,6,12$, and $24 \mathrm{~h}$, and repeatability was measured via analyzing the peak area and retention time of five random chosen samples treated using the same procedure. RSDs were used to depicted Variations. The Precision of the measured samples was between $1.20-3.10 \%$. The stability was determined to be ranged from 0.4 to $0.7 \%\left(t_{R}\right)$ and from 1.1 to $3.2 \%$ (peak area). For the reproducibility, the RSDs of $t_{R}$ and peak area varied from 0.15 to $0.67 \%$ and from 1.15 to $3.6 \%$, respectively. Therefore, the selected method was of great reliability for profiling of $A$. argyi samples.

\subsection{HPLC Fingerprint Establishment and Similarity Analysis, Calibration of References in HPLC Fingerprint}

We have four reference compounds (rutin, apigenin, 
jaceosidin, and eupatilin). However, the study didn't identify other characteristic chromatographic peaks for fresh $A$. argyi samples except for those of jaceosidin and eupatilin. Howerver, the peak of jaceosidin was quite weak, and the peak of eupatilin seemed featuring, so eupatilin was chosen as a reference $\left(t_{R}=6.7 \pm 1\right.$ min, Peak 1 in Figure 2) and the content of which has been determined in our study.

\subsection{Analysis and Comparison of HPLC Fingerprints}

The chromatographic fingerprints of 52 germplasm sources of $A$. argyi from different locations (Figure 1) are presented in Figure 3. Generally speaking, characteristic peak selection was based on a certain criterion that peaks observed in each chromatogram of different samples should be well separated under the optimized chromatographic conditions and have relatively large areas in different profiles. The reference fingerprint (marked as $\mathrm{R}$ in Figure 3, the upper one) was established as the median of 52 chromatograms to identify and evaluate the quality of $A$. argyi, and 14 peaks were extracted as characteristic common peaks. To facilitate identification and analysis, the whole chromatogram was divided into three parts, namely part $\mathrm{A}\left(t_{R} 0-15.0 \mathrm{~min}\right)$, part $\mathrm{B}\left(t_{R} 15.0-30.0 \mathrm{~min}\right)$ and part $\mathrm{C}\left(t_{R} 30.0-48.0 \mathrm{~min}\right)$. Among all of these peaks, that of part $\mathrm{C}$ has obvious characteristics, which allowed part $\mathrm{C}$ to be used as a featuring area of $A$. argyi. On the contrary, the fingerprints of part A and B were quite variable, most of the chromatographic peaks and peak areas dependent on sample origin, which could be used as the basis for the identification of this medicinal material.

\subsection{Effects of Sample Origin on HPLC Fingerprints}

The Tangyin County of Henan Province is the main area of northern $A$. argyi herb production since the ancient time, which has excellent quality. Therefore, we systematically investigated the fingerprints of $A$. argyi produced in Tangyin, and compared it with the sources of Ningbo, Qichun and Anguo productions to provide guidelines for the selection of high-quality germplasm for the Chinese medicine industry and agriculture use. For uniform comparison, unprocessed fresh leaves were used for fingerprint analysis (Figure 4), which revealed that samples produced in Tangyin was different from those produced elsewhere in part $\mathrm{C}$ of the fingerprints. All samples contain eupatilin, a typical flavonoid compound whose peak presented at around $t_{R} 6.7 \mathrm{~min}$ in part A, and the part B seems indistinguishable for all of the four samples. In part $\mathrm{C}$, Ningbo $A$. argyi has larger peaks $(\mathrm{C} 1, \mathrm{C} 4, \mathrm{C} 7$ and $\mathrm{C} 10)$ than other samples, especially $\mathrm{C} 1$ is the highest of all. In case of Qichun and Anguo samples, peaks in part $\mathrm{C}$ were very similar. However for Tangyin samples, peaks $\mathrm{C} 10$ and $\mathrm{C} 11$ were not so obvious, whereas a minor peak $\mathrm{C} 10$ was observed for samples of other origins. There are small peaks $\mathrm{C} 2$, C3, C5 for Ningbo sample and peak C11 is not observed for other samples.

\subsection{Similarity Analysis of Fingerprints of Different Samples}

China has a lot of Injections prepared from herbs, to control the quality of the final products the government required that all herbal Injection products should be evaluated the similarity, which can be determined by analyzing a battery of original chromatographic data. Similarity analysis was therefore performed to evaluate the differences between fresh $A$. argyi samples. From a comprehensive view in Figure 3 , the chromatographic profiles of the tested samples quite similar, although the retention times and peak areas were kind of variable. The similarity of all samples ranged from 0.17 to 0.967 . The similarity values of 25 samples exceeded 0.9 . However, low similarity values of $<0.6$ observed for samples 36 and 45 suggested that the compositions of these samples might be different from those of samples with high similarity values. According to Huang et al [19], eupatilin was quite stable and can be used as the identification control component. For the content of eupatilin, Ningbo $A$. argyi was the highest in the four typical fresh samples. However, A. argyi has abundant secondary metabolites, the highest content of one characteristic compound does not equal to the best quality of this herb. Therefore, the complex pharmacological activity research and the correlation analysis of the material basis of the compounds is necessary to tell which sample was really of good quality.

\subsection{Chemometrics Analysis}

Hierarchical clustering analysis (HCA) is the analysis process of grouping a set of physical or abstract objects into multiple classes composed of similar objects. It's an important human behavior. The goal of cluster analysis is to collect data for classification on the basis of similarity. It has been widely applied to fingerprint analysis [20-21]. To assess the resemblance and differences between different $A$. argyi samples as a whole, HCA of the $52 \mathrm{~A}$. argyi samples was performed based on the relative areas of characteristic peaks.

The results (Figure 5 obviously indicated that most Henan samples (except S14 and S8) were clustered together. Qichun (S34) and Ningbo (S51) samples were different from others, and S26 (Puyang, Henan) was classified into a separate group. The similarity between S25 (Shaoyang, Hunan) and other samples exceeded 0.9 , but these two sample groups were still treated as part by cluster analysis. HCA results provided further references for the quality evaluation of $A$. argyi.

Principal component analysis (PCA) is a statistical method that allows us to summarize the big data by means of a smaller set of "summary indices" that can be more easily visualized, which is an unsupervised multivariate data analysis approach [23]. In this research, PCA was employed to analyze the relationships between the $52 \mathrm{~A}$. argyi samples of different germplasm resources, projecting them to low-dimensional space to observe subtle differences. The resulting score plot is presented in Figure 6. It shows clearly that sample S26 (Puyang, Henan) and S48 (Xi'an, Shannxi) falls out of the 
oval area.

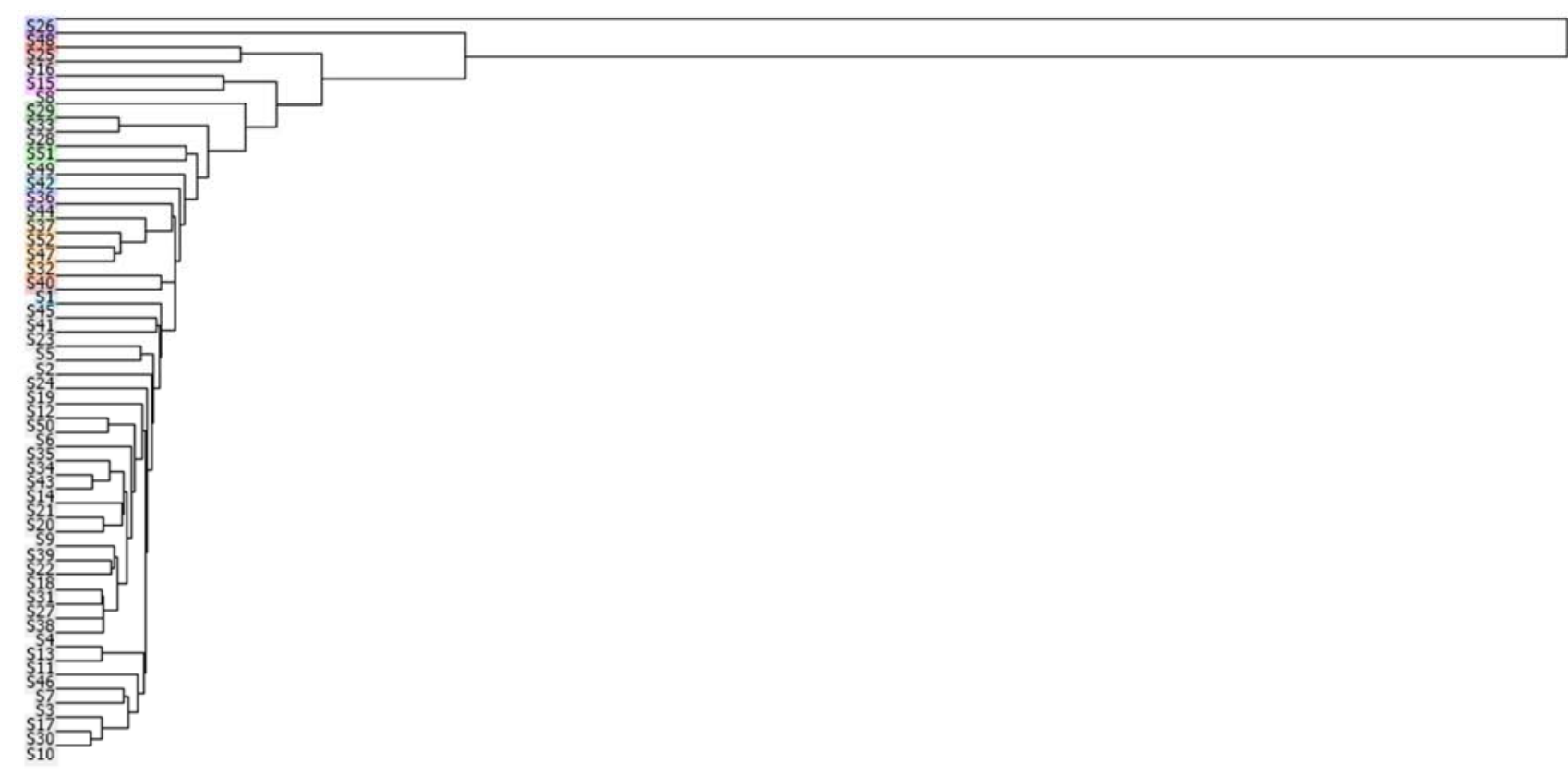

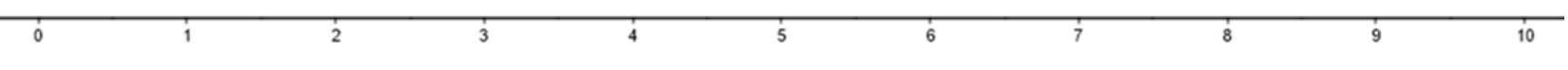

Figure 5. HCA dendrogram for the 52 A. argyi samples.

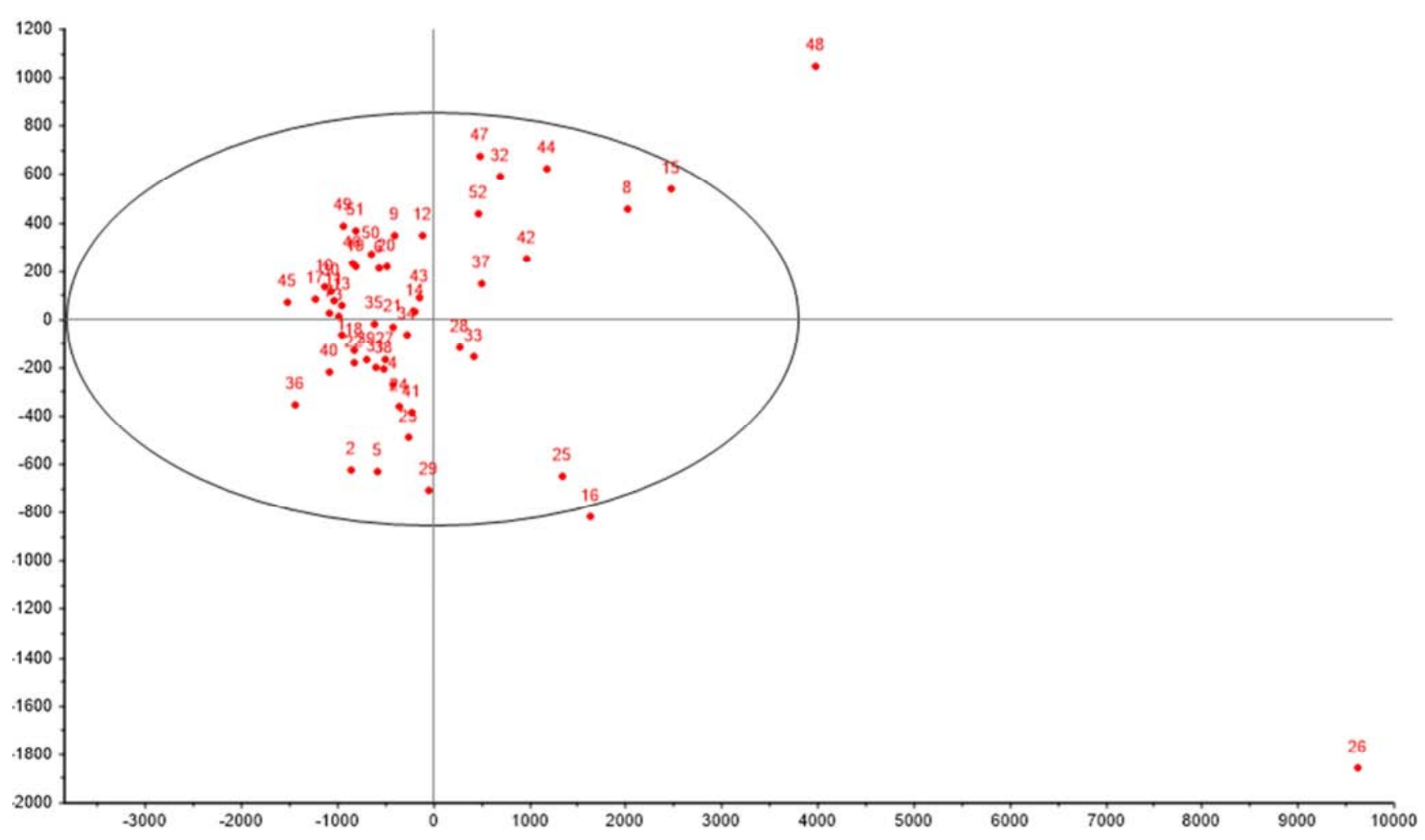

Figure 6. PCA results for the $52 \mathrm{~A}$. argyi samples. 3.9. Establishment of a standard HPLC fingerprint of A. argyi from Henan province

Featuring fingerprint selection. By studying the fingerprints of 52 samples of $A$. argyi, we selected 10 samples (S5, 10, 13, 15, 17, 27, 30, 37, 39, 46) of typical $A$. argyi germplasm resources collected in different locations of Henan province and constructed their standard fingerprints.

Relative areas of common peaks. After comparison and analysis, 14 common peaks of $A$. argyi were identified (Figure 7), with peaks 5,8 and 12 accounting for more than $5 \%$ of the 
total peak area. The total peak area of each batch of chromatographic fingerprints exceeded $90 \%$, in compliance with fingerprinting requirements.

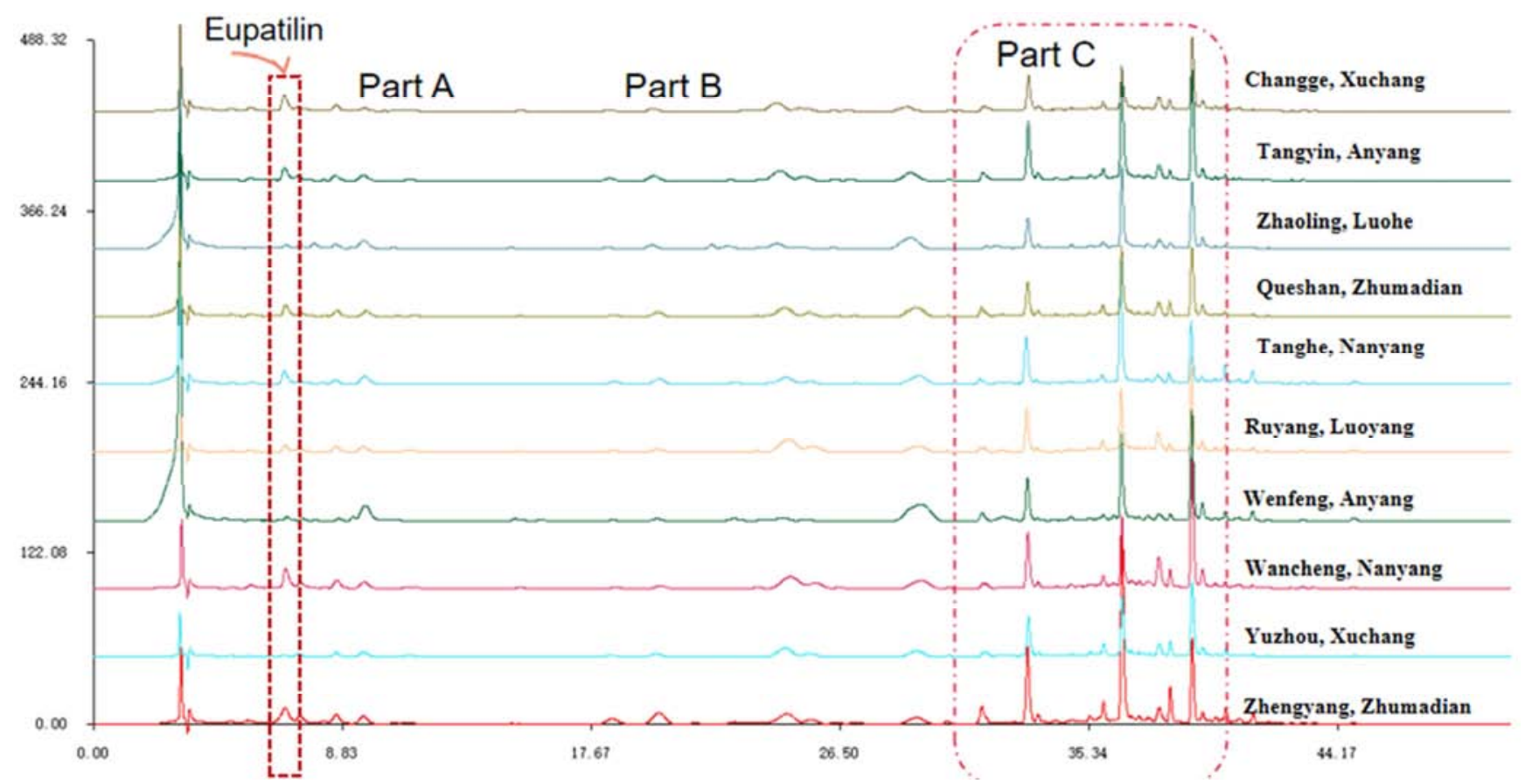

Figure 7. HPLC fingerprints of A. argyi from different geographical origins in Henan province.

Generation of common patterns. The fingerprints of 10 batches of $A$. argyi produced in different locations of Henan province were automatically established and matched by using the software called Similarity Evaluation System. First, each spectrum was introduced to the system, and then the reference spectrum was set by selecting the one produced in Tangyin county, multi-point calibration and automatic matching were applied. In all 10 batches, 14 common peaks were observed between 5 and $48 \mathrm{~min}$. Then, the peak areas and retention time of these 14 common peaks were measured. The median method was applied to generate the simulated common chromatogram.

\subsection{Eupatilin Content Difference}

The flavone eupalitin is the characteristic component in the fresh leaves of $A$. argyi. In order to check the presence of eupatilin in all the 52 samples, the content of which has been determined. As shown in Table 1, the fresh sample from Puyang germplasm source had the highest content $(27.64 \mu \mathrm{g} / \mathrm{g})$ and from Luzhou, Sichuan province had the lowest content $(3.50 \mu \mathrm{g} / \mathrm{g})$. However, the Puyang sample doesn't seem to be normal because it falls out the major group of $A$. argyi based on the PCA, indicating it might be an adulterated resource or wrong species. Other fresh germplasm samples origin from Quzhou, Zhejing (10.16 $\mu \mathrm{g} / \mathrm{g})$ and Jinan, Shandong $(7.35 \mu \mathrm{g} / \mathrm{g})$ have high content of eupatilin too. As mentioned earlier, flavone content is only one factor of the substantial basis of the bioactivity of $A$. argyi. Therefore, the sample of highest eupatilin content doesn't mean the overall quality is the best.

\section{Conclusions}

Fingerprint as a quality control method of herbal medicine and their preparations has become an international consensus, a variety of fingerprint control technology system in line with the characteristics of traditional Chinese medicine is being studied and established. The United States FDA allows chromatographic fingerprints in herbal supplements, and WHO also stipulated in the guidelines for the evaluation of herbal medicine in 1996 that if the active ingredient of the herb is not clear, chromatographic fingerprints can be provided to prove the consistency of product quality. The European community guideline on the quality of herbal medicines also states that it is not sufficient to determine the stability of the quality of an active ingredient, as the whole of the herb and its preparation is the active substance. The application of fingerprint aims to solve the problems of quality detection and quality difference between batches of medicinal plants with complex ingredients and unclear active ingredients. The study of fingerprint of traditional Chinese medicine is an innovative research work, which applies modern separation methods and instrumental analysis techniques to the quality control of TCM in order to establish a new quality control method. In addition, the establishment and analysis of the fingerprint of each traditional Chinese medicine are also remarkably innovative.

Herbs exposed to different geographical environment may have different chemical content and quality. A HPLC fingerprint and quantitative analysis method was established 
for the quality evaluation of fresh $A$. argyi leaves from different germplasm sources. The changes of chemical fingerprints due to various environmental factors or genotypes were verified by systematic comparison of the chromatograms of 52 samples. HPLC is a comprehensive multivariate data set due to the complexity of the composition of traditional Chinese medicine, so small differences between very similar chromatograms may be overlooked. Therefore, the chemical pattern recognition method was used to classify $A$. argyi leaves reasonably and identify them effectively, and the influence of geographical location and germplasm resources on the identification of $A$. argyi leaves was discussed. This information is expected to help identify and evaluate the quality of $A$. argyi because of its great potential in food, pharmaceutical and value-added products. The research results will provide theoretical basis for supporting provenance technology and standardized ecological cultivation technology, and have demonstration and guiding significance for other authentic medicinal materials in Henan province, and have good social, ecological and economic benefits.

\section{Acknowledgements}

The authors of this study express their gratitude to Doctoral Research Foudation of Anyang Institute of Technology (BSJ2021031, YPY2020028), Key Technologies R \& D Program of Henan Province (212102311024) for financial supports.

\section{References}

[1] Otsuka, K., Shoji, J., Takido, M., Cho, S. A Pictorial Encyclopedia of Chinese Medical Herbs, Chuokoran-Sha Inc.: Tokyo, Japan, 1992.

[2] Li, J., Zhao, B. Safety concerns about the application of moxa. J. Acupunct. Tuina Sci. 8, 145-148 (2010).

[3] Mei, Q., Chen, X., Xiang, L., Liu, Y., Su, Y., Gao, Y., Dai, W. Dong, P., Chen, S. DNA barcode for identifying folium Artemisiae argyi from counterfeits. Biol. Pharm. Bull. 39, 1531-1537 (2016).

[4] Huang, L. Q., Qiu, L. Revision to origin of northern Artemisia argyi in Compendium of Materia Medica (Bencao gangmu). China J. Chin. Mater. Med. 39, 4887-4890 (2014).

[5] Chinese Pharmacopoeia Committee. Pharmacopoeia of People's Republic of China, First Div. 2015 ed. (Beijing: China Chemical Industry Press, 2015).

[6] Song, X., Wen, X., He, J., Zhao, H., Li, S., Wang, M., Phytochemical components and biological activities of Artemisia argyi, J. Funct. Foods 52, 648-662 (2019).

[7] Lan, X. Y., Zhang, Y., Zhu, L. B., Liu, D. H., Huang, X. Z., Zhou, L., Kang, L. P. Research progress on chemical constituents from Artemisiae Argyi Folium and their pharmacological activities and quality control. China J. Chin. Mater. Med. 45, 4017-4030 (2020).
[8] Zheng, X., Deng, C., Song, G., Hu, Y. Comparison of essential oil composition of Artemisia argyi leaves at different collection times by headspace solid-phase microextraction and gas chromatography-mass spectrometry. Chromatographia 59, 729-732 (2004).

[9] Lan, M. B., Zhang, Y. H., Zheng, Y., Yuan, H. H., Zhao, H. L., Gao, F. Antioxidant and immunomodulatory activities of polysaccharides from Moxa (Artemisia argyi) leaf. Food Sci Biotechnol. 19, 1463-1469 (2010).

[10] Samuel, A. J., Kalusalingam, A., Chellappan, D. K., et al. Ethnomedical survey of plants used by the Orang Asli in Kampung Bawong, Perak, West Malaysia. J. Ethnobiol. Ethnomed. 6, 20137098 (2010).

[11] Guo, L., Jiao, Q., Zhang, D., Liu, A. P., Wang, Q., Zheng, Y. G. Quality evaluation of Artemisiae argyi folium based on fingerprint analysis and quantitative analysis of multicomponents. China J. Chin. Mater. Med. 43, 977-984 (2018).

[12] Wang, Y., Wu, H., Yang Y., Tian, J. Wang, X. Identification of argy wormwood leaf by HPLC fingerprint. Pharma. Info. 4, 35-41 (2015)

[13] Yu, F., Sun, L., Zhou, Q. Analysis on fingerprint of Artemisiae argyi folium by HPLC. China. Arch. Trad. Chin. Med. 30, 2432-2434 (2012).

[14] Wang, Y., Wu, H., Yang, Y., Wang, X., Zhang, W. UPLC Fingerprints of Artemisae argyi folium. China J. Exper. Trad. Med. Form. 13, 50-52 (2015).

[15] Wang, Y., Zhuang, G., Zhang, X., Geng, R. Analysis and comparison of HPLC fingerprints of wanai and Artemisiae argyi from thirty-one growing areas by multistatistical. Chin. Trad. Patent Med. 40, 653-658 (2018).

[16] Lu, Y., Ma, Q., Fu, C., Chen, C., Zhang, D. Quality evaluation of Corydalis yanhusuo by high-performance liquid chromatography fingerprinting coupled with multicomponent quantitative analysis. Sci. Rep. 10, 4996 (2020).

[17] Qin, K. M. et al. Quality assessment of raw and processed Arctium. lappa L. through multicomponent quantification, chromatographic fingerprint, and related chemometric analysis. J. Sep. Sci. 38, 1491-1498 (2015).

[18] Schorr, K, Da Costa, F. B. Quantitative determination of enhydrin in leaf rinse extracts and in glandular trichomes of Smallanthus sonchifolius (Asteraceae) by reversed-phase high-performance liquid chromatography. Phytochem. Anal. $16,161-165$ (2005).

[19] Huang, X. Z., Kang, L. P, Gao, L., Zhang, Y., Guo, L. P., Huang, L. Q. Quantative analysis of eupatilin and jaceosidin in folium of Artemisia argyi from different areas in China by RP-HPLC based on ancient medicine books. China J. Chin. Mater. Med. 42, 3504-3508 (2017).

[20] Kong, W., Zhao, Y., Xiao, X., Jin, C. \& Li, Z. Quantitative and chemical fingerprint analysis for quality control of Rhizoma Coptidischinensis based on UPLC-PAD combined with chemometrics methods. Phytomedicine 16, 950-959 (2009).

[21] Lu, F. et al. Studies on chemical fingerprints of Siraitia grosvenorii fruits (Luo Han Guo) by HPLC. J. Nat. Med. 66, 70-76 (2012). 
[22] Liu, J. et al. Chemical fingerprinting of wild germplasm resource of Ophiopogon japonicus from Sichuan basin, China by RP-HPLC coupled with hierarchical cluster analysis. Anal. Lett. 43, 2411-2423 (2010).

[23] $\mathrm{Wu}, \mathrm{Q}$. Y. et al. Chromatographic fingerprint and the simultaneous determination of five bioactive components of Geranium carolinianum L. water extract by high performance liquid chromatography. Int. J. Mol. Sci. 12, 8740-8749 (2011).
[24] Zhai, W. M. Similarity Evaluation System for Chromatographic Fingerprint of TCM, Version 2004A. (China Pharmacopoeia Committee: Beijing, China. 2004).Huffman, J., Gerber, R., Du, L (2010). Recent advancements in the biosynthetic mechanisms for polyketide derived mycotoxin. Biopolymers, 93, 764-776. 